\title{
LA CONTRIBUCIÓN DE LOS FACTORES POLÍTICOS, EDUCATIVOS Y ECONÓMICOS EN LA SEGURIDAD CIUDADANA EN LIMA METROPOLITANA
}

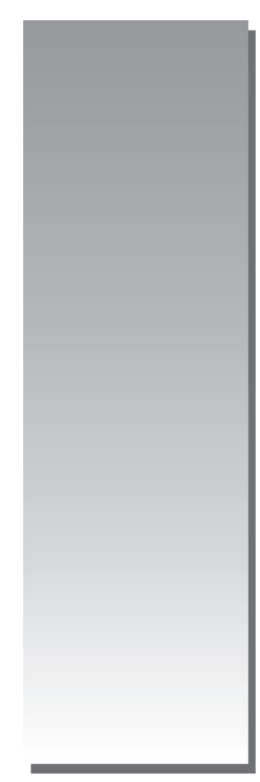

The CONTRIBUtion OF THE POLITICAL, EDUCATIONAL AND ECONOMIC FACTORS OF THE CiTIZEN SECURITY IN LIMA

\author{
Manuel Jorge Espinoza Altamirano* \\ manuelespinoza123@yahoo.es
}

[RECEPCIÓN: ABRIL 2016 / CONFORMIDAD: MAYO 2016]

\section{RESUMEN}

El presente artículo busca establecer la contribución de los factores político, educativo y económico en la seguridad ciudadana de Lima Metropolitana puesto que actualmente el principal problema existente es la inseguridad ciudadana tanto a nivel distrital como a nivel nacional. Para el desarrollo de esta investigación se utilizó el método cualitativo, cuantitativo, correlacional y transversal. El tipo de estudio es analítico, descriptivo y aplicativo. Se emplearon herramientas estadísticas y de recolección de datos de fuentes primarias tales como cuestionarios y entrevistas, así como la obtención de datos a través de fuentes secundarias. Como fuentes primarias los datos obtenidos fueron de los 43 distritos de Lima Metropolitana y cuya muestra total corresponde a 384 personas encuestadas respecto a los factores de orden político, educativo y económico y su incidencia en la seguridad ciudadana en cada localidad distrital. Los resultados expresaron que los factores políticos educativos y económicos constituyen variables importantes que determinarán el nivel de seguridad ciudadana en Lima Metropolitana.

Como conclusiones y propuestas del trabajo de investigación es importante considerar que la seguridad ciudadana constituye una política de estado que es de responsabilidad y compromiso del gobierno, quien debe delinear las políticas y estrategias para enfrentar en forma efectiva los principales problemas de delincuencia, violencia, percepción de inseguridad ciudadana y la corrupción. Esta última existente en los diferentes niveles de orden interno y administración de justicia tales como la Policía Nacional, El Poder Judicial y el Ministerio Publico en Lima Metropolitana, así como en el País. Así mismo debería tomarse en cuenta las recomendaciones efectuadas a mayor detalle tanto a nivel de orden político, educativo y económico propuesto en el presente trabajo de investigación.

Palabras clave: Seguridad ciudadana; delincuencia; violencia; corrupción; percepción de inseguridad; política; educación; economía.

\section{ABSTRACT}

The present research is aimed to establish the contribution of the political, educational and economic factors in the Citizen security in Lima as the main existing problem the insecurity at the district and national level. For the development of this research the qualitative, quantitative, correlational and

\footnotetext{
Magister en Ciencias Administrativas con mensión en Gestión Empresarial UNMSM Doctor en Ciencias Administrativas, contador, auditor y consultor empresarial
} 
transversal method was used. The type of study is analytical, descriptive and application. For the development of this research used statistical tools and data collection from primary sources such as questionnaires and interviews and data collection through secondary sources. As primary sources of data were the 43 districts of Lima and whose total sample is 384 respondents regarding factors of political, educational and economic order and its impact in the security citizen in every district town. The results show that education and economic and political factors are important variables that determine the level of Citizen Security.

Conclusions and proposals of the research is important to consider that public safety is a state policy that is the responsibility and commitment of the government, which should outline policies and strategies to deal effectively with the major problems of crime, violence, perception of insecurity and corruption. The latter existing at different levels of internal order and administration of justice such as the National Police, the Judiciary and the Public Ministry in Lima and in the country. Also it should take into account the recommendations made in more detail at the level of political, educational and economic order proposed in this research.

Keywords: Citizen security; crime; violence; corruption; perception of insecurity; politics; education; economy.

\section{INTRODUCCIÓN}

De acuerdo al Plan Nacional De Seguridad Ciudadana, aprobado por el Consejo Nacional De Seguridad Ciudadana (CONASEC) refrendado por Decreto Supremo $N^{\circ} 012-2013-I N$, señala que la seguridad ciudadana es un fenómeno social complejo, multidimensional y multicausal, que debe ser abordado desde diversos aspectos en forma simultánea tales como aspectos políticos, económicos, educativos, entre otros.

De acuerdo a información del INEI publicado en el diario el Correo del 11 de Octubre del 2013, el $90 \%$ de personas viven con percepción de inseguridad. Así mismo se revela que el $40.4 \%$ de personas fue víctima de algún hecho delictivo y del universo de personas que han sido víctimas de este hecho, solo el $12.8 \%$ denunció.

Según Fruhling y Tulchin (2005), entre los factores sociales más destacados están la desigualdad en los ingresos, el acceso a las armas de fuego, los efectos de la guerra, la debilidad de los controles institucionales en particular la fragilidad de los sistemas judiciales y policiales, las normas culturales $\mathrm{y}$, a la vez, los niveles de pobreza y la historia de violencia. Las normas sociales y culturales también son un determinante básico del comportamiento.

\section{OBJETIVOS:}

Objetivo general:

En el presente artículo de investigación se busca determinar los factores que influyen en la seguridad ciudadana de Lima Metropolitana,
Objetivos específicos:

1. Determinar si los componentes del factor político influye en la seguridad ciudadana de Lima Metropolitana.

2. Determinar si los componentes del factor educativo influye en la seguridad ciudadana de Lima Metropolitana.

3. Determinar si los componentes del factor económico influyen en la seguridad ciudadana de Lima Metropolitana.

\section{MARCO TEÓRICO \\ Seguridad}

El término de seguridad de acuerdo al Diccionario de la Real Academia Española proviene del latin securitas que significa cualidad de seguro y la palabra seguro se deriva del latin securus que significa libre y exento de todo peligro, daño o riesgo.

Henry Fayol, Ingeniero Minero, conocido como padre de teoría clásica de la administración, en el año 1919 establece la seguridad como una de las seis funciones básicas de la empresa, función relacionada con la protección y preservación de los bienes de las personas.

Para el Programa de las Naciones Unidas para el Desarrollo (PNUD, 1994), la seguridad es uno de los elementos que definen la calidad de vida de las personas y el el desarrollo humano debe estar indisolublemente ligado a la seguridad humana, que tiene como propósito proteger al individuo frente a amenazas de distinta naturaleza: de- 
sastres naturales, criminalidad, enfermedades y epidemias, hambre, pobreza extrema, dictaduras y totalitarismo.

Diversas teorías de desarrollo humano y tratados internacionales de derechos humanos reconocen el derecho a la seguridad e integridad corporal como un derecho humano fundamental de los individuos. El Pacto Internacional de Derechos Civiles y Políticos (1966) establece en su artículo 9 que "todo individuo tiene derecho a la libertad y a la seguridad personales" y la Declaración Universal de Derechos Humanos (1948) reconoce el "derecho a la vida, a la libertad y a la seguridad de la persona" (Art. 3).

\section{Factor económico}

Según David Begg, Fisher y Rudiger Dornobusch (2006), la economía es el estudio de cómo la sociedad decide qué, cómo y para quien producir. $\mathrm{Al}$ resaltar el papel de la sociedad la propia definición sitúa a la economía dentro del conjunto de las ciencias sociales. A si mismo indica que el objeto de estudio en la economía es el comportamiento humano en la producción, el intercambio y el uso de bienes y servicios.

Según Mc Eachern William A. (1998), la economía es el estudio de cómo la gente decide asignar sus recursos escasos para producir intercambiar y consumir bienes y servicios en un intento de satisfacer sus necesidades ilimitadas. Los recursos son los insumos utilizados para producir los bienes y servicios que son escasos. Se dividen en cuatro grandes categorías: tierra, trabajo, capital y habilidad empresaria (conocimiento).

\section{Factor educativo}

Giddens Anthony (2009) define a la educación como una institución social, que permite o fomenta la adquisición de habilidades, conocimientos y la ampliación de los horizontes personales.

Para Durkheim (1925), considera que la educación desarrolla un papel importante en la socialización de los niños ya que en concreto con el aprendizaje de la historia, esto adquiere una comprensión de los valores comunes de la sociedad, que sirven para unir a una multitud de individuos separados. Estos valores comunes incluyen las creencias religiosas y morales y un sentido de autodisciplina. Para este autor la educación permite a los niños interiorizar las normas sociales que contribuyen al funcionamiento de la sociedad.
Según Bowles y Gintis (1976) la estructura de las relaciones sociales del sistema educativo no solo habitúa al estudiante a la disciplina del lugar del trabajo, sino que además desarrolla los tipos de comportamiento personal, el modo de presentarse uno mismo, la propia imagen y las identificaciones de clase social que son los ingredientes cruciales para la educación del empleo. Las relaciones sociales que se producen en el marco educativo (entre administradores y profesores, profesores y estudiantes y entre los estudiantes y sus propias tareas) reproducen las divisiones jerárquicas del trabajo.

\section{Factor político}

La política de acuerdo a Light (1995) es el proceso social mediante el cual las personas obtienen y pierden el poder. El poder es la habilidad de ejercer el control sobre estas personas a pesar de sus resistencias. El poder colectivo o habilitante es el poder de un grupo para lograr que las cosas se hagan con la cooperación y la división laboral. Así mismo este autor considera que el Estado es una entidad abstracta y está conformado por la suma de instituciones que se especializan en ejercer el poder y la autoridad incluye los tribunales, la legislatura, el poder ejecutivo, las fuerzas armadas y otros cuerpos oficiales.

\section{Inseguridad ciudadana}

La inseguridad está influenciada por múltiples creencias, actitudes, valores y experiencias que pueden o no tener relación con el delito. En este sentido, la satisfacción con la seguridad pública está determinada, tanto por la percepción subjetiva de seguridad y certidumbre en la vida del ciudadano, como por su confianza en la eficacia de las instituciones, principalmente la Policía según Ruiz Díaz (1997).

La inseguridad ciudadana se define como el temor a posibles agresiones, asaltos, secuestros, violaciones, de los cuales podemos ser víctimas según El PNUD (2006: 35).Hoy en día, es una de las principales características de todas las sociedades modernas, y es que vivimos en un mundo en el que la extensión de la violencia se ha desbordado en un clima generalizado de criminalidad.

\section{Seguridad ciudadana}

Zavaleta y Bielefeltd (2013) define La seguridad ciudadana a la necesidad de mantener y poten- 
ciar las relaciones interpersonales en el marco de la ley y la cultura, expresadas en el respeto al derecho ajeno bajo la norma, para lo cual tienen presencia un conjunto de instituciones públicas (municipio, justicia, cárcel) y sociales (universidades, medios de comunicación, defensores de derechos humanos).

Miraglia y Serrano (2011) señala que la seguridad ciudadana es un enfoque integral que requiere la participación de una amplia gama de actores. Es por eso que esta ofrece información útil para otros actores claves que participan en estos esfuerzos, tales como gobiernos nacionales y locales, organizaciones de la sociedad civil y las mismas comunidades.

El PNUD (2006: 35) define la seguridad ciudadana como la condición personal, objetiva y subjetiva, de encontrarse libre de violencia o amenaza de violencia o despojo intencional (violento o pícaro) por parte de otros.

\section{Método de la Investigación}

Para el desarrollo de esta investigación se utilizó el método cualitativo, cuantitativo, correlacional y transversal. El tipo de estudio es analítico, descriptivo y aplicativo.

Las investigaciones en Ciencias Sociales se ocupan de la descripción de las características que identifican diferentes elementos y componentes, y su interrelación según Hernández Roberto (2014).

Para el desarrollo del presente estudio, se desarrollaran muestras no probabilísticas para determinar el tamaño de muestro del sector público con relación a la seguridad ciudadana de Lima Metropolitana, así como determinar la selección de expertos en este tema. Así mismo se aplicó el método cuantitativo para determinar la población de estudio, el tamaño de la muestra, selección de la muestra.

La población en estudio está conformada por personas mayores de 18 años de todo los distritos de Lima Metropolitana de acuerdo a la información del INEI (2013) respecto al censo de la población por distritos. Así mismo instituciones que directamente están ligados a la Seguridad ciudadana, tales como el Ministerio de Interior, Municipalidades distritales entre otros. De acuerdo a la información publicada por el INEI, la Población de Lima Metropolitana es de 6, 630, 000 habitantes.

\section{Resultados \\ Factor Político}

1) ¿Considera Ud. que existe un nivel de compromiso del gobierno local (Municipalidad) de su distrito respecto a la seguridad ciudadana?

Un aspecto muy importante en la seguridad ciudadana es que la población considere que existe compromiso por parte del Gobierno Local en la lucha contra la inseguridad ciudadana, en este sentido, podemos observar, que sólo el $13 \%$ de los encuestados conocen afirman que existe compromiso del Gobierno Local, en tanto, el 87\% de los encuestados considera que el Gobierno Local no está comprometido con el mejoramiento de la Seguridad Ciudadana.

Tabla № 1. Nivel de compromiso del gobierno local respecto a la seguridad ciudadana. Lima, Setiembre 2015.

\begin{tabular}{ccccc}
\hline & Frecuencia & Porcentaje & $\begin{array}{c}\text { Porcentaje } \\
\text { acumulado }\end{array}$ \\
\hline Válido & $\begin{array}{l}\text { Muy satisfecho } \\
\text { Satisfecho }\end{array}$ & 44 & 11,4 & 13,0 \\
\hline $\begin{array}{lccc}\text { Medianamente } \\
\text { satisfecho }\end{array}$ & 84 & 21,8 & 34,8 \\
\hline $\begin{array}{llcc}\text { Poco satisfecho } \\
\text { Nada satisfecho }\end{array}$ & 133 & 34,5 & 69,4 \\
\hline Total & 385 & 30,6 & 100,0 \\
\hline
\end{tabular}

Fuente: Elaboración propia. Encuesta realizada - Setiembre 2015

2) ¿La atención brindada por la Policía Nacional en su distrito es oportuna cuando es requerida?

La labor de la Policía Nacional debe de actuar de manera oportuna cuando se requiere de sus servicios, para lo cual, podemos observar que sólo un 7,5 \% de los encuestados considera que el PNP actúa de manera oportuna en su distrito, y por otro lado, un $92,5 \%$ opina que la Policía Nacional del Perú no actúa de manera oportuna. Según lo mencionado, podemos decir que este aspecto afecta la confianza que pueda tener el ciudadano respecto a la Policía Nacional del Perú como una entidad que debe imponer orden y autoridad. 
Tabla № 2. La Policía Nacional respecto a su atención oportuna. Lima, Setiembre 2015.

\begin{tabular}{ccccc}
\hline & Frecuencia & Porcentaje & $\begin{array}{c}\text { Porcentaje } \\
\text { acumulado }\end{array}$ \\
\hline \multirow{2}{*}{ Válido } & Muysatisfecho & 6 & 1,6 & 1,6 \\
\cline { 2 - 5 } & $\begin{array}{l}\text { Satisfecho } \\
\text { Medianamente }\end{array}$ & 23 & 6,0 & 7,5 \\
\cline { 2 - 5 } & satisfecho & 92 & 23,9 & 31,4 \\
\cline { 2 - 5 } & Pocosatisfecho & 146 & 37,9 & 69,4 \\
\cline { 2 - 5 } & Nadasatisfecho & 118 & 30,6 & 100,0 \\
\hline & 385 & 100,0 & \\
\hline
\end{tabular}

Fuente: Elaboración propia. Encuesta realizada- Setiembre 2015

3) ¿La atención brindada por el Serenazgo en su distrito es oportuna cuando es requerida?

Podemos apreciar en el cuadro, que un 15,8 de los encuestados opina que el Serenazgo brinda atención oportuna cuando es requerida, frente a un $84,2 \%$ que manifiesta que la atención no es oportuna.

Tabla № 3. Serenazgo respecto a su atención oportuna. Lima, Setiembre 2015.

\begin{tabular}{|c|c|c|c|c|}
\hline & & Frecuencia & Porcentaje & $\begin{array}{l}\text { Porcentaje } \\
\text { acumulado }\end{array}$ \\
\hline \multirow{6}{*}{ Válido } & Muy satisfecho & 11 & 2,9 & 2,9 \\
\hline & Satisfecho & 50 & 13,0 & 15,8 \\
\hline & $\begin{array}{l}\text { Medianamente } \\
\text { satisfecho }\end{array}$ & 94 & 24,4 & 40,3 \\
\hline & Pocosatisfecho & 127 & 33,0 & 73,2 \\
\hline & Nadasatisfecho & 103 & 26,8 & 100,0 \\
\hline & Total & 385 & 100,0 & \\
\hline
\end{tabular}

Fuente: Elaboración propia. Encuesta realizada - Setiembre 2015

4) ¿Considera Ud. que existe corrupción en el Poder Judicial?

El Perú es uno de los países Latinoamericanos con más corrupción en la labor de las entidades que deben velar por el bienestar de todos los ciudadanos; de esta manera, podemos observar que una mayoría del $94 \%$ considera que en el Poder Judicial existe corrupción, frente a un $6 \%$ que considera lo contrario. Por lo antes mencionado podemos mencionar que el sistema de gobierno del país necesita cambios si se quiere brindar seguridad ciudadana.
Tabla № 4. Corrupción en el Poder Judicial. Lima, Setiembre 2015.

\begin{tabular}{ccccc}
\hline & Frecuencia & Porcentaje & $\begin{array}{c}\text { Porcentaje } \\
\text { acumulado }\end{array}$ \\
\hline \multirow{2}{*}{ Válido } & \multicolumn{1}{c}{ Sí } & 362 & 94,0 & 94,0 \\
\cline { 2 - 5 } & No & 23 & 6,0 & 100,0 \\
\cline { 2 - 5 } & Total & 385 & 100,0 & \\
\hline
\end{tabular}

Fuente: Elaboración propia. Encuesta realizada - Setiembre 2015

5) ¿Considera Ud. que existe corrupción del Ministerio Público (fiscalía)?

Continuando con el análisis de la corrupción en el Estado, podemos observar que un $91,4 \%$ de los encuestados cree que existe corrupción en el Ministerio Público, frente a un 8,6\% que opina lo contrario. De aquí podemos señalar que, si los ciudadanos no tienen confianza en la labor de las entidades que velan por la seguridad ciudadana y creen que existe corrupción en los entes que establecen las sanciones a los que infringen la Ley; entonces, no nos debe sorprender que exista un alto índice de inseguridad ciudadana en Lima Metropolitana.

Tabla № 5. Corrupción en el ministerio público. Lima, Setiembre 2015.

\begin{tabular}{ccccc}
\hline & & Frecuencia & Porcentaje & $\begin{array}{c}\text { Porcentaje } \\
\text { acumulado }\end{array}$ \\
\hline \multirow{2}{*}{ Válido } & Sí & 352 & 91,4 & 91,4 \\
\cline { 2 - 5 } & No & 33 & 8,6 & 100,0 \\
\cline { 2 - 5 } & Total & 385 & 100,0 & \\
\hline
\end{tabular}

Fuente: Elaboración propia. Encuesta realizada - Setiembre 2015

6) Durante los últimos doce meses, ¿Usted, un familiar o amigo fue víctima de algún delito?

Respecto a los actos delincuenciales, podemos observar que casi un $70 \%$ de los encuestados manifiesta haber sufrido algún delito en los últimos doce meses; de acuerdo a estos resultados podemos evidenciar que la inseguridad ciudadana vivida actualmente es un problema grave y que debe ser apaciguado en el menor tiempo posible. 
Tabla № 6. Personas que han sido víctimas de algún delito en los últimos 12 meses. Lima, Setiembre 2015.

\begin{tabular}{ccccc}
\hline & & Frecuencia & Porcentaje & $\begin{array}{c}\text { Porcentaje } \\
\text { acumulado }\end{array}$ \\
\hline \multirow{3}{*}{ Válido } & Sí & 265 & 68,8 & 68,8 \\
\cline { 2 - 5 } & No & 120 & 31,2 & 100,0 \\
\cline { 2 - 5 } & Total & 385 & 100,0 & \\
\hline
\end{tabular}

Fuente: Elaboración propia. Encuesta realizada - Setiembre 2015

7) ¿Cree usted que el estado está realmente comprometido con mejorar el nivel de la seguridad ciudadana?

Tabla № 7. El Estado y su compromiso con la seguridad ciudadana. Lima, Setiembre 2015.

\begin{tabular}{ccccc}
\hline & & Frecuencia & Porcentaje & $\begin{array}{c}\text { Porcentaje } \\
\text { acumulado }\end{array}$ \\
\hline \multirow{3}{*}{ Válido } & Sí & 57 & 14,8 & 14,8 \\
\cline { 2 - 5 } & No & 328 & 85,2 & 100,0 \\
\cline { 2 - 5 } & Total & 385 & 100,0 & \\
\hline
\end{tabular}

Fuente: Elaboración propia. Encuesta realizada- Setiembre 2015

Para culminar el factor político, se quiere conocer si los ciudadanos creen que el Estado está comprometido en mejorar el nivel de seguridad ciudadana; de esta manera, podemos observar que sólo un $14.8 \%$ de los encuestados manifiestan que el Estado está realmente comprometido con la seguridad ciudadana; frente a un $85.2 \%$ de los encuestados quienes piensan lo contrario. De aquí se puede evidenciar que las políticas no están orientadas a un real compromiso del Estado a mejorar la Seguridad.

\section{Factor Educativo}

8) ¿Considera adecuada la calidad de educación impartida en los centros educativos de su distrito?

Continuando con el análisis de la calidad de educación impartida; podemos observar que un $10,4 \%$ de los encuestados manifiestan que la educación impartida en los centros educativos es de calidad, frente a un $89,6 \%$ el cual opina lo contrario. De aquí podemos seguir sustentando el poco esfuerzo en la educación por parte del Estado, o cual está afectado otros aspectos en Lima Metropolitana y en el Perú.
Tabla № 8. Opinión respecto a la Calidad de educación en los centros educativos. Lima, Setiembre 2015.

\begin{tabular}{clccc}
\hline & Frecuencia & Porcentaje & $\begin{array}{c}\text { Porcentaje } \\
\text { acumulado }\end{array}$ \\
\hline \multirow{2}{*}{ Válido } & Muy suficiente & 5 & 1,3 & 1,3 \\
\cline { 2 - 5 } & Suficiente & 35 & 9,1 & 10,4 \\
\cline { 2 - 5 } & $\begin{array}{l}\text { Medianamente } \\
\text { suficiente }\end{array}$ & 155 & 40,3 & 50,6 \\
\cline { 2 - 5 } & & 38,4 & 89,1 \\
\cline { 2 - 5 } & Poco suficiente & 148 & 10,9 & 100,0 \\
\cline { 2 - 5 } Insuficiente & 42 & 100,0 & \\
\hline
\end{tabular}

Fuente: Elaboración propia. Encuesta realizada - Setiembre 2015

9) ¿Cree Ud. que en los centros educativos de su distrito se imparte una educación formativa en valores (respeto, solidaridad, verdad, paz, justicia, equidad, responsabilidad, entre otros)?

Tabla № 9. Los centros educativos respecto a la educación formativa en valores. Lima, Setiembre 2015.

\begin{tabular}{ccccc}
\hline & Frecuencia & Porcentaje & $\begin{array}{c}\text { Porcentaje } \\
\text { acumulado }\end{array}$ \\
\hline \multirow{4}{*}{ Válido } & Muybueno & 9 & 2,3 & 2,3 \\
\cline { 2 - 5 } & Bueno & 52 & 13,5 & 15,8 \\
\cline { 2 - 5 } & Regular & 195 & 50,6 & 66,5 \\
\cline { 2 - 5 } & Malo & 96 & 24,9 & 91,4 \\
\cline { 2 - 5 } & Muy malo & 33 & 8,6 & 100,0 \\
\hline & Total & 385 & 100,0 & \\
\hline
\end{tabular}

Fuente: Elaboración propia. Encuesta realizada - Setiembre 2015

10)¿Cree Ud. Que los padres de familia en su distrito imparten valores en la formación de sus hijos y son modelo de vida de estos últimos?

Los valores impartidos dentro del ambiente familiar tienen un carácter imprescindible para el desarrollo apropiado y fructífero de los adolescentes en vías de definir su camino en la adultez. En este sentido podemos observar, que un $32,7 \%$ de los encuestados manifiesta que los padres de familia de su distrito imparten de manera adecuada los valores en la familia; frente a un $67,3 \%$ el cual considera que no se imparte de la manera adecuada. De aquí se puede señalar que este es un punto de partida que debe ser mejorado para un desarrollo a largo plazo de la seguridad ciudadana, puesto que las acciones son guiadas por la actitud y la formación de las personas, este aspecto debe ser seriamente considerado. 
Tabla № 10. Los padres de familia respecto a la formación con valores de sus hijos. Lima, Setiembre 2015.

\begin{tabular}{|c|c|c|c|c|}
\hline & & Frecuencia & Porcentaje & $\begin{array}{l}\text { Porcentaje } \\
\text { acumulado }\end{array}$ \\
\hline \multirow{6}{*}{ Válido } & Muybueno & 14 & 3,6 & 3,6 \\
\hline & Bueno & 112 & 29,1 & 32,7 \\
\hline & Regular & 175 & 45,5 & 78,2 \\
\hline & Malo & 57 & 14,8 & 93,0 \\
\hline & Muymalo & 27 & 7,0 & 100,0 \\
\hline & Total & 385 & 100,0 & \\
\hline
\end{tabular}

Fuente: Elaboración propia. Encuesta realizada - Setiembre 2015

\section{Factor Económico}

11)¿Cree usted que los ciudadanos de su distrito tienen la capacidad económica para cumplir con sus necesidades básicas (alimentación, vivienda, vestidos, seguridad, salud, entre otros)?

Tabla № 11. Los ciudadanos y su capacidad económica para cumplir con sus necesidades básicas. Lima, Setiembre 2015.

\begin{tabular}{clccc}
\hline & Frecuencia & Porcentaje & $\begin{array}{c}\text { Porcentaje } \\
\text { acumulado }\end{array}$ \\
\hline \multirow{2}{*}{ Válido } & Muysuficiente & 10 & 2,6 & 2,6 \\
\cline { 2 - 5 } & Suficiente & 68 & 17,7 & 20,3 \\
\cline { 2 - 5 } & $\begin{array}{l}\text { Medianamente } \\
\text { suficiente }\end{array}$ & 178 & 46,2 & 66,5 \\
\cline { 2 - 5 } & & 25,7 & 92,2 \\
\cline { 2 - 5 } & Pocosuficiente & 99 & 7,8 & 100,0 \\
\cline { 2 - 5 } Insuficiente & 30 & 100,0 & \\
\cline { 2 - 5 } & Total & 385 & & \\
\hline
\end{tabular}

Fuente: Elaboración propia. Encuesta realizada - Setiembre 2015

La capacidad económica la cual va de la mano del nivel de desempleo deben ser considerados en un análisis de seguridad ciudadana debido a su influencia en la misma. De aquí se puede observar que sólo un $20.3 \%$ de los encuestados considera que los ciudadanos de su distrito tienen la capacidad suficiente o muy suficiente para cumplir con sus necesidades básicas, frente a un 79.7\% el cual ha manifestado lo contrario. Es así como podemos señalar que sin capacidad económica para la sostenibilidad familiar, no se podrán realizar cambios significativos en la mejora de la seguridad ciudadana.
12)¿Cree usted que la carencia económica de las personas en su distrito son una de las causas principales de la violencia y la delincuencia?

Analizando las causas principales de la violencia y la delincuencia, podemos observar que un $39,7 \%$ de los encuestados considera que está muy seguro o seguro de que la carencia económica es una de las causas principales, un 31,9\% manifiesta estar mediamente seguro al respecto, y un $28,3 \%$ cree que no es un de las causas principales. De aquí podemos se señalar que la carencia económica influye en gran medida en la seguridad ciudadana.

Tabla № 12 . La carencia económica como una de las causas principales de la violencia y delincuencia. Lima, Setiembre 2015.

\begin{tabular}{llccc}
\hline & Frecuencia & Porcentaje & $\begin{array}{c}\text { Porcentaje } \\
\text { acumulado }\end{array}$ \\
\hline \multirow{2}{*}{ Válido } & Muy seguro & 60 & 15,6 & 15,6 \\
\cline { 2 - 5 } & Seguro & 93 & 24,2 & 39,7 \\
\cline { 2 - 5 } & $\begin{array}{l}\text { Medianamente } \\
\text { seguro }\end{array}$ & 123 & 31,9 & 71,7 \\
\cline { 2 - 5 } & & 18,2 & 89,9 \\
\cline { 2 - 5 } Unpocoseguro & 70 & 10,1 & 100,0 \\
\hline Muy inseguro & 39 & 100,0 & \\
\hline
\end{tabular}

Fuente: Elaboración propia. Encuesta realizada - Setiembre 2015

13)¿Cree Ud. que exista oportunidad de empleo en su distrito?

Tabla № 13. Existencia de oportunidad de empleo en los distritos de Lima Metropolitana. Lima, Setiembre 2015.

\begin{tabular}{ccccc}
\hline & & Frecuencia & Porcentaje & $\begin{array}{c}\text { Porcentaje } \\
\text { acumulado }\end{array}$ \\
\hline Válido & Si & 163 & 42,3 & 42,3 \\
\cline { 2 - 5 } & No & 222 & 57,7 & 100,0 \\
\cline { 2 - 5 } & Total & 385 & 100,0 & \\
\hline
\end{tabular}

Fuente: Elaboración propia. Encuesta realizada - Setiembre 2015

Se deduce que la falta de oportunidad de empleo en los Distritos de Lima Metropolitana contribuye a incrementar la inseguridad ciudadana en la localidad. 


\section{DISCUSIÓN}

1. De acuerdo a la interpretación de la Tabla №1 Nivel de compromiso del gobierno local respecto a la seguridad ciudadanía se interpreta que el gobierno local no está comprometido con el mejoramiento de la seguridad ciudadana de su Distrito.

2. De acuerdo a la interpretación de la Tabla № 2 la Policía Nacional del Perú, no actúa de manera oportuna afectando la confianza del ciudadano en cuanto al desempeño de la autoridad policial en su localidad.

3. La interpretación de los resultados presentados en la tabla № 3 respeto a la atención brindada por el Serenazgo en su distrito, manifiesta claramente al ciudadano que la atención que brinda el Serenazgo no es oportuna y no acude con prontitud cuando es requerido. Situación esta que genera insatisfacción sobre la calidad de sus servicios, consecuentemente afecta el nivel de seguridad ciudadana.

4. Respeto a la interpretación de resultados presentado en la tabla №4 Corrupción en el poder judicial, puede inferirse que existe un alto nivel de corrupción en el Poder Judicial, hecho que denota una deficiente administración de justicia plagada por la corrupción, consecuentemente constituye una causa importante de la inseguridad ciudadana y falta de confianza en este Poder.

5. La tabla 5. Corrupción en el Ministerio Público permite inferir que un alto porcentaje de los ciudadanos considera que existe corrupción en el Ministerio Público. El Ministerio Publico como organismo constitucional autónomo del Estado Peruano como parte de la Administración de la Justicia no defiende la legalidad de los intereses y derechos de la ciudadanía debido a la corrupción existente causando insatisfacción y propiciando la inseguridad ciudadana en Lima Metropolitana.

6. Del análisis de resultado de la tabla 6, personas que han sido víctimas en los últimos 12 meses puede inferirse que un $70 \%$ de los encuestados fueron pasibles de algún delito, hecho que constituye un problema grave que genera un constante temor e inseguridad en el ciudadano de la localidad, no permitiéndole desarrollar con tranquilidad y seguridad sus actividades cotidianas
7. De acuerdo al resultado de la Tabla 7 El Estado y su compromiso con la Seguridad Ciudadana, puede interpretarse que los ciudadanos en un $85.2 \%$ de Lima Metropolitana creen que el Estado no está seriamente comprometido en mejorar la Seguridad Ciudadana, al mostrar políticas y acciones débiles que no permiten contrarrestar la inseguridad existente. Esta actitud pasiva es caldo de cultivo para el incremento de la inseguridad ciudadana.

8. De acuerdo al resultado de la Tabla 8. Opinión respecto a la calidad de educación en los centros educativos, puede interpretarse que el $89.6 \%$ indica que la calidad impartida en los centros educativos de Lima Metropolitana y en el Perú no es el adecuado y que existe poco esfuerzo en mejorar el nivel educativo existente, afectando directamente la buena formación educativa y de competencia que debe tener el estudiante, y de este modo garantizar un mejor desempeño en el futuro en cualquier actividad que tenga que desarrollar.

9. De acuerdo al resultado de la Tabla 9. Los centros educativos respecto a la educación formativa en valores, puede interpretarse que actualmente los centros educativos de los distritos de Lima Metropolitana no imparten una educación formativa basada en valores que permita internalizarse en los estudiantes y de este modo cambiar, mejorar su conducta y actitud frente a los retos, desafíos y responsabilidades que deberá asumir en el futuro, de este modo, estará contribuyendo a elevar el nivel de seguridad ciudadana.

10.De acuerdo al resultado de la Tabla 10. Los Padres de Familia respecto a la formación con valores de sus hijos, se interpreta que un porcentaje considerable del $67.3 \%$ de los encuestados indica que los valores impartidos por los padres de familia en sus hogares, no son los más apropiados ni fructíferos para los hijos, hecho que en la adultez o enel futuro afectaría seriamente en su conducta y quehacer como ciudadano.

11.De acuerdo al resultado de la Tabla 11 . Los ciudadanos y su capacidad económica para cumplir con sus necesidades básicas, puede inferirse que un $79.7 \%$ de los ciudadanos no cuenta con la capacidad suficiente para cubrir sus necesidades básicas para la sostenibilidad de su familia, generándoles impotencia y capa- 
cidad para afrontar día a día el sustento de su familia, hecho que podría llevarlo a delinquir y consecuentemente elevar la inseguridad reinante en su localidad o trasladarse a otras localidades para cometer actos delictivos.

12.De acuerdo al resultado de la Tabla 12 . La carencia económica como una de las causas principales de la violencia y delincuencia, puede interpretarse que una de las causas principales de la violencia y la delincuencia es la carencia económica existente en ciertos sectores de las localidades distritales, afectando la seguridad ciudadana.

13. De acuerdo al resultado de la Tabla 13. Existencia de oportunidad de empleo en los distritos de Lima Metropolitana, puede inferirse que la falta de oportunidad de empleo o trabajo en los distritos de Lima Metropolitana constituye una de las causas que genera la inseguridad ciudadana existente en las localidades distritales, situación que podría mejorarse generando mayores inversiones empresariales, consecuentemente mayor empleo y oportunidad de desarrollo.

\section{CONCLUSIONES}

De la investigación efectuada se arribó a las siguientes conclusiones:

1. Dentro del aspecto político considerando la importancia de la seguridad ciudadana un $87 \%$ de los encuestados consideran que no existe compromiso con el mejoramiento de la seguridad ciudadana por parte del gobierno local.

2. Es muy importante destacar la importancia de la labor de la Policía Nacional del Perú (PNP) en cuanto a atención oportuna de sus servicios y se observa en la presente investigación que en 92.5\% de los encuestados opinan que la PNP no actúa de manera oportuna ante un requerimiento afectando la confianza al ciudadano puesto esta institución debe imponer orden y autoridad. El nivel de confianza frente a su desempeño es muy bajo puesto que el $78.8 \%$ opina que la PNP no les genera confianza.

3. Como resultado del análisis respecto a la corrupción se concluye: considera un $82.6 \%$ de los encuestados que existe corrupción en la PNP. El 94\% de los encuestados consideran la existencia de corrupción en el Poder Judicial y el $91.4 \%$ consideran que existe corrupción en el Ministerio Publico.
4. Existe un alto nivel de victimización producto de la delincuencia, puesto que un $70 \%$ de las personas encuestadas opinan que fueron víctimas de un delito. Este hecho evidencia un alto nivel de inseguridad. Este nivel de victimización tiene una relación lógica con la falta de vigilancia permanente por parte de la PNP. Así mismo se observa la falta de vigilancia permanente por parte del Serenazgo.

5. La educación es el pilar fundamental en la formación de la persona y/o ciudadano. El nivel y calidad de educación brindada generalmente se refleja en nivel de la seguridad ciudadana ya sea en una localidad o en un país y para ello es importante la capacitación continua de los docentes.

6. La formación en valores no solo recae en los maestros o educadores. Los padres de familia son los principales formadores de valores en hijos, generalmente los hijos son el fiel reflejo de la conducta de los padres. Esta formación de valores tienen gran incidencia en la seguridad ciudadana.

7. Se hace imprescindible impartir una educación de calidad teniendo como referente los estándares internacionales de calidad aplicado al sector de educación. Esta formación posibilitaría que los egresados de estos centros educativos tengan la capacidad de insertarse laboralmente o emprender una actividad empresarial. De este modo la brecha existente de desempleo en los distritos podría disminuir contribuyendo a mejorar la seguridad ciudadana reduciendo los niveles de violencia y delincuencia. Así mismo es importante el acceso igualitario a la educación para reducir estos niveles de criminalidad.

8. Existe en promedio un alto nivel de desempleo en los distritos de Lima Metropolitana, así como la carencia económica para cumplir con sus necesidades básicas o primarias (alimentación, vivienda, vestidos, seguridad entre otros), situación que puede llevar la persona a cometer delitos con el fin de cubrir sus necesidades y la sostenibilidad de su familia. La falta de oportunidad de empleo, la desigualdad económica y el nivel de pobreza existente lleva a la carencia económica de los ciudadanos en Lima Metropolitana constituyendo una de las causas principales de la violencia y la delincuencia. 


\section{RECOMENDACIONES}

1. Se recomienda a los líderes de los Gobiernos locales plantear políticas y estrategias de sensibilización, desarrollar un compromiso incondicional con los ciudadanos de su distrito y buscar la mejora en forma continua en el tema de la seguridad ciudadana, que principalmente brindan a través del serenazgo.

2. La Policía Nacional del Perú (PNP), como institución responsable de la seguridad interna en el país, dentro de los distritos de su jurisdicción debe tener la capacidad de respuesta adecuada y oportuna frente a cualquier requerimiento de su competencia, imponiendo orden, respeto y autoridad, a fin de obtener la confianza en su localidad. Así mismo, se recomienda que esta institución, a través de sus superiores, evalúe permanentemente el nivel de desempeño de los policías con el fin de mejorar o retroalimentar las carencias o deficiencias de este personal y, de este modo, se hagan acreedores de la confianza y respeto de la comunidad, que actualmente está en serio cuestionamiento.

3. Es política y responsabilidad del Estado establecer lineamientos y estrategias que permitan reducir el alto nivel de corrupción en la Policía Nacional del Perú, el Poder Judicial y el Ministerio Público. Se recomienda establecer y desarrollar procesos de selección de personal por competencias a través de perfiles personales y profesionales preestablecidos, además ser evaluados psicológicamente para conocer sus rasgos de personalidad, conducta y vocación de servicio del nuevo personal.

4. Así mismo, debe efectuarse un proceso de evaluación y control de carácter permanente en cada una de estas instituciones, debiendo establecerse un sistema de quejas que tenga una amplia difusión. La atención de estas quejas deben ser efectuadas con celeridad, equidad y justicia, estableciendo penas severas y efectivas a quienes cometan actos de corrupción o de cualquier otro delito.

5. Teniendo en cuenta que la Educación constituye un pilar fundamental en el desarrollo del ciudadano, y consecuentemente en el desarrollo de la localidad y del país, se recomienda establecer en los planes curriculares de los diferentes niveles de educación, la educación cognitiva por competencias, así como la educación formativa en valores, que en el mediano y largo plazo contribuirá a fortalecer la seguridad ciudadana.

6. Como política del Estado, los Gobiernos nacional, regionales y locales deben establecer políticas y estrategias para promover a través de todos los medios existentes - consejeros, psicólogos, educadores, medios de difusión, entre otros- la importancia y la práctica de valores. En un futuro tendríamos ciudadanos con mejor calidad de vida y probos que contribuirían a mejorar la seguridad ciudadana.

7. Se recomienda que el sistema educativo nacional, regional y local, en sus diferentes niveles, debe propender a desarrollar una educación basada en estándares internacionales de calidad, debiendo promoverse la acreditación. Así mismo, debe impartirse una educación formativa en valores en todos los niveles. De aplicarse esta política educativa, posibilitaría a los egresados insertarse con mayor facilidad en el campo laboral o empresarial reduciendo de este modo la brecha actual de desempleo. Además, debe promoverse el acceso igualitario a la educación sin distinción de raza, credo y condición económica para generar mayores oportunidades laborales y de desarrollo en la sociedad, contribuyendo así a fortalecer la seguridad ciudadana.

8. Es recomendable que dentro de su política de Estado el gobierno promueva el desarrollo económico a través de las inversiones extranjeras y nacionales, lógicamente estas inversiones constituirían fuente de generación de empleo, desarrollar y mejorar la infraestructura del país. Así mismo el gobierno debe promover y financiar el emprendimiento de micro y pequeñas empresas en el País para generar auto empleo y empleo. Bajo estos conceptos y su ejecución podrían los ciudadanos cubrir sus necesidades básicas o primarias y mejorar su condición económica y la de su familia generando mayor oportunidad de empleo y así contribuir en la reducción de la brecha de la desigualdad económica y el nivel de pobreza existente. 


\section{REFERENCIAS BIBLIOGRÁFICAS}

Fruhling, Hugo y Joseph TULCHIN (2005) Crimen y Violencia en América Latina. Seguridad Ciudadana, Democracia y Estado. Bogotá: Fondo de Cultura Económica.

Begg, David; Fischer, Stanley; Dornbusch, Rudiger y Fernández, Andrés. (2006). Economía. 8a ed. Madrid, McGraw-Hill.

Mc Eachern William A. Economía una introducción contemporánea (1998).International Thomson Editores. España.

Giddens Anthony (2009). Sociología. Alianza Editorial. Madrid

Bowles, S y H. Gintis (1976). Schooling in Capitalist America: Educational Reform and Contradictions of Economic Life, New York, Basic Books.
Light Donald (1995). Sociología. México: McGrawHill.

Ruiz Díaz, Carmen (1997). Los españoles y la inseguridad ciudadana. Colección Opiniones y Actitudes №12. Madrid: Centro de Investigaciones Sociológicas. Paper.

Zavaleta y Bielefeltd (2013). Los retos de la seguridad ciudadana, Estudios de Seguridad y Defensa № 1. México. Paper.

Miraglia Paula, Serrano-Berthet Miraglia (2011). Alianzas públicas y privadas para la Seguridad Ciudadana. Instituto Sou da Paz y el Banco Mundial. Paper.

Programa de las Naciones Unidas para el Desarrollo (2013) Seguridad Ciudadana con rostro humano: diagnóstico y propuestas para América Latina. Estados Unidos. Paper. 